\title{
Personalised medicine in paediatrics: individualising treatment in children with rare neurological diseases
}

\author{
Maurizio Scarpa • Adriana Ceci • Rosella Tomanin • \\ Pierpaolo Mincarone $\cdot$ David Begley
}

Received: 24 March 2011 / Accepted: 11 April 2011 / Published online: 1 May 2011

(C) European Association for Predictive, Preventive and Personalised Medicine 2011

\begin{abstract}
The development of personalised medicine is of considerable importance for paediatric patient populations, and represents a move away from the use of treatment dosages based on experience with the same compounds in adults. Currently, however, we know little about developmental pharmacogenomics and, although many biomarkers are available for clinical research use, there have been few applications in the management of paediatric diseases. This paper reviews where we are in the journey towards achieving paediatric personalised medicine and describes a group of diseases requiring such an approach. The personalised medicine approach is particularly relevant for the treatment of rare childhood diseases, and the group of life-threatening neurological disorders known as lysosomal storage diseases represents a potential study population. The genetic bases of these disorders are generally well defined, there is the potential for diagnosis at birth or prenatally, and there are a range of therapeutic options available or under development.
\end{abstract}

Keywords Paediatric population $\cdot$ Neurological impairment · Rare disease - Biomarker · Pharmacogenomic . Personalised medicine

M. Scarpa $(\bowtie) \cdot R$. Tomanin

Department of Pediatrics, University of Padova,

Via Giustiniani 3,

35128 Padova, Italy

e-mail: maurizio.scarpa@unipd.it

\section{A. Ceci $\cdot$ P. Mincarone}

Pharmacological Research Foundation Gianni Benzi,

Bari, Italy

D. Begley

King's College London,

London, UK

\section{Introduction}

Today, clinical research is strongly regulated with the aim of ensuring that clinical trials are well designed and conducted in an ethical manner. In Europe, several guidelines, directives and regulations pertaining to clinical trials have been released by the European Parliament, Council and Commission [1-3]. One in particular, Directive 2001/ 20/EC, establishes specific provisions regarding the conduct of clinical trials of medicinal products on human subjects and recognises the principles of good clinical practice [1]. In line with this, many medicines have been developed based on the results of well-designed, randomised, controlled clinical trials, and these treatments are currently used in a range of patient groups, offering considerable clinical and economic utility in healthcare. That said, however, it is widely acknowledged that not all patients respond in the same way to a given therapy and, as a result, the current methodological approach will require updating in future to take account of individual patient characteristics.

For many adult and some childhood conditions, biomarkers that reliably reflect inter-individual variability in disease expression and that also correlate with or predict outcomes have already been discovered among the genes, mRNA, proteins and metabolites found in the body. Polymorphisms in the human leukocyte antigen (HLA), for example, have been identified as risk factors for severe adverse drug reactions (ADRs): these include the association between HLA-B*1502 and carbamazepine-induced skin necrolysis and Stevens-Johnson syndrome [4], as well as the association between HLA-B*5701 and hypersensitivity reactions to the HIV drug, abacavir $[5,6]$. Screening for both of these alleles is recommended before starting the respective treatment and, in the case of HLA-B*5701, 
screening prior to abacavir initiation has been suggested to produce cost savings $[6,7]$. In future, it is expected that such biomarkers will be detected more readily thanks to the new high-throughput technologies and powerful analytical approaches employed within the fields of pharmacogenomics, proteomics, metabolomics and other so-called 'omics'.

This type of genetic knowledge may ultimately be included within the drug development process where biomarkers will serve as identifiers of treatment response. As a result, it will become important to include population screening early in the clinical trial development phase in order to establish which subpopulations receiving an experimental drug are most/least likely to benefit from, or to experience adverse reactions associated with, that treatment. Refinement of this approach will lead to more targeted patient recruitment and should reduce the likelihood of new therapies failing at the clinical trial stage. Most importantly, it should reduce the risk of unsuccessful treatments or undesired secondary adverse effects in clinical practice. Whether pharmacodiagnostic (theranostic) applications can simplify the selection of drugs for clinical testing, and help to develop more focused clinical trials that provide enhanced knowledge about the effects of a drug in humans within shorter time frames, are currently being explored by various organisations such as the National Institutes of Health (NIH) [8].

\section{The use of biomarkers in paediatrics}

The use of biomarkers as indicators of genetic factors affecting drug response is of particular interest for children. Historically, despite the need for treatments for conditions affecting children, for ethical, economic and methodological reasons, there have been few clinical trials in young populations $[9,10]$. As a result, off-label drug use in paediatric patients has been relatively common $[9,11,12]$. An example of this is the fact that metabolic capacity is different in children and adults. Hepatic glucoronidation activity of the UDP-glucuronosyltransferase (UGT) family of enzymes that are needed for the elimination of certain xenobiotic and endogenous substances, for instance, is lower in children than adults which may have implications for drug detoxification [13]. UGT activity towards bilirubin at birth, for example, is approximately $1 \%$ of the level observed in adults [14], although activity increases rapidly and reaches levels equivalent to those in adults 14 weeks after birth [15]. Total UGT activity has been reported to develop to adult levels by 20 months of age; however, that age at which enzymes reach adult levels of activity may not be universally indicative of in vivo drug clearance [16]. This study showed that intrinsic hepatic clearance of the antipsychotic drug trifluoperazine did not appear to reach adult levels until 18.9 years of age despite UGT being maximally active well before this time. These types of infancy/childhood-specific developmental changes are clearly complex and likely underlie some of the variability in drug response seen in neonates and children. Failing to take the potential impact of these differences on drug action into account places children at risk of ADRs.

In recent years there has in fact been a rise in the number of paediatric drug safety and efficacy studies, as well as changes in drug labelling for young patients that show unique paediatric doses are often necessary [17, 18]. Furthermore, some diseases that occur in children either do not develop in adults or have a different effect/ prevalence in adults, making it necessary to develop drugs specifically for children. Despite the clear need for individualised medicine for paediatric patient populations, we still know very little about developmental pharmacogenomics and there have been few applications of biomarkers in the management of paediatric disease [19]. Results of a literature search conducted by the Task force in Europe for Drug Development for the Young (TEDDY) European Network of Excellence demonstrated that throughout the world more than $50 \%$ of pharmacogenomic/pharmacogenetic research activities in children during the last 10 years were related to predisposition, which means the investigation of the correlation between genetic traits and the probability of or susceptibility for a given pathology or disease [20]. These types of exploratory studies provide little or no insight into mechanisms of disease or drug action, and their results cannot be used to improve medical practice or to support therapeutic solutions. Only a small number of polymorphisms that are linked to therapeutic response have been studied in children and, even when studies demonstrate an impact on treatment response, their use in clinical practice remains limited. This is highlighted by the fact that very few FDA drug label amendments for pharmacogenomic testing refer to paediatric populations [9].

A major consideration that has arisen from analyses of publications on pharmacogenetics and pharmacogenomics is that studies often show contradictory or inconsistent results. This is particularly the case for studies in paediatric populations which, by definition, generally have limited patient numbers. A systematic review and field synopsis of pharmacogenetic studies in general that was conducted in 2009 suggested that the limited translation of pharmacogenetic research into practice may be explained by the preponderance of reviews over primary research, small sample sizes, a mainly candidate gene approach, the use of surrogate markers, an excess of nominally positive or truly positive associations and paucity of meta-analyses [21]. The small sample sizes, coupled with the more frequent evaluation of common rather than rare variants, the use of 
surrogate outcome measures rather than more clinically relevant outcomes, and subgroup analysis with multiple hypothesis testing, suggest that only a proportion of the positive associations reported are in fact genuine [21]. Clear analysis of the situation is further complicated by the use of different research methods across studies, the lack of both standardised outcome measures and study replication, and the low level of consideration given to potential covariates such as co-morbidities. Other investigators have similarly raised concerns about the need to improve the quality of studies and prevent false-positive associations [19]. In conclusion, while the new 'omics' techniques offer great promise for the identification of biomarkers of drug response, these studies are subject to many of the same pitfalls that apply in general to paediatric and other clinical trials.

Notwithstanding the limited experience and considerations above, some exciting examples of the usefulness of biomarkers in clinical practice can be derived from recent published literature. Childhood disorders in which pharmacogenetic studies have most commonly been conducted include acute lymphoblastic leukaemia, attention-deficit hyperactivity disorder, growth hormone deficiency, juvenile idiopathic arthritis, atopic dermatitis and asthma (see [19, $22,23]$ for reviews). A good example of a childhood condition where there will be benefits from the use of pharmacogenetics is acute lymphoblastic leukaemia (ALL). Current treatment for patients with ALL results in eventfree survival greater than $75 \%$ for most patients; however, approximately $25 \%$ fail to respond to treatment [24]. One of the reasons for treatment failure is the development of toxicity due to the absence or presence of only low levels of enzymes that metabolise chemotherapies. Thiopurines (azathioprine, mercaptopurine and thioguanine), for example, are often used to treat ALL and their safety has been shown to be related to thiopurine methyltransferase (TPMT) genotype. Genotypes resulting in reduced TPMT enzyme activity have been shown to be associated with dose-related side effects/toxic events to thiopurines in children with ALL [25-27]. Thus, TPMT genotype may be used to help choose the appropriate dose of thiopurine for an individual child. In the US, the drug labels for azathioprine and 6-mercaptopurine now give the FDA recommendation for TPMT testing [9]. Although this biomarker is not yet reliably assessed in children within Europe, because TPMTdeficiency can now be easily detected, this knowledge should lead to improvements in the safety of treatment in children receiving thiopurine chemotherapy. The recent publication of Clinical Pharmacogenetics Implementation Consortium Guidelines for TPMT genotyping and thiopurine dosing may also facilitate the routine incorporation of TPMT genotyping in clinical practice for paediatric patients receiving these compounds [28].

\section{What is available and what is needed to develop personalised medicines?}

There are several pharmacogenomic/pharmacogenetic networks already in existence that aim to help collect, coordinate or communicate information about genetic determinants of drug response. In Europe, for example, there is the European Network for Pharmacogenetics (http://www.epr-network.org/), in the US there is the NIH Pharmacogenomics Research Network (http://www.nigms. nih.gov/Initiatives/PGRN), in Canada, the Canadian Pharmacogenomics Network for Drug Safety (CPNDS), and there is also the International HapMap project (http:// hapmap.ncbi.nlm.nih.gov/) that represents a partnership of researchers and funding agencies from several countries. Each of these networks is distinct; however, the activities of the CPNDS illustrate how the establishment of networks may play an important role in the development of personalised medicine. The CPNDS is an active surveillance network that collects information on ADRs, and operates predominantly within Canadian paediatric teaching hospitals $[9,29]$. The aim of the network is to improve drug safety by identifying genetic markers that can predict which patients are likely to experience specific serious ADRs, as well as those who may experience decreased therapeutic response to a drug. To help determine the role of genetic variations in the development of ADRs, the network has created a database of clinical ADRs and a bio-bank of DNA and other samples. Among other examples, this approach has already proven useful by contributing to the development of a research protocol to validate reports of a potential association between genetic variations in enzymes in the CYP and UGT families in breastfeeding women taking codeine and the development of codeine-induced central nervous system (CNS) depression in their babies [30].

The development of databases containing information about genomic positions and bio-banks containing tissue samples from large numbers of individuals also make it possible to conduct genome-wide association studies [19, 31]. Rather than selecting candidate genes, this type of investigation can look at thousands of single nucleotide polymorphisms across the genome in one investigation which may represent an important approach for studying complex, common diseases where multiple genetic variations may contribute to drug response.

As mentioned previously, despite the fact that many biomarkers are currently available for use within the clinical research setting, few have been integrated into paediatric clinical practice. In addition, the validation process of such markers in children remains an issue. As a consequence, it is important to encourage the establishment of paediatric bio-banks, as well as the connection of existing bio-banks through intelligent informatics platforms 
in order to share data, integrate results and promote the use of biomarkers in drug development and clinical trials. Furthermore, the uptake of biomarkers within the drug development process requires investment and research on the part of the pharmaceutical industry. As of 2007, very few pharmaceutical companies indicated ongoing pharmacogenomic or pharmacogenetic-related research in children [20]. However, more recently an investigation performed by Tufts suggests a growing interest and investment in this area. Their report shows that $12 \%-50 \%$ of current clinical pipelines of the companies interviewed involve personalised medicines [32]. Thus, although at present paediatric drug development and utilisation cannot benefit fully from pharmacogenetic/pharmacogenomic findings there is hope for the future. Further efforts are needed in order to reduce the gap between promise and application.

Particular actions that have to be undertaken in order to reduce the existing gaps include:

0 the identification of the most promising research approaches for use in paediatric populations from among those based on 'omics' sciences;

$\circ$ the development of model-based methodologies for clinical trials in children based on the use of 'omics'derived biomarkers;

$\circ$ the initiation and completion of pilot studies designed with the goal of developing paediatric personalised medicines in a specific sector such as oncology, respiratory disease, neurodegenerative disease or infective disease.

The development of personalised medicine is of relevance for all paediatric patient groups, and in particular for those populations that are generally deprived of drugs when the traditional clinical trial approach is applied. Examples of populations for whom the personalised medicine approach will be particularly important are neonates and children with rare diseases.

\section{Choice of a model disease to study personalised medicine in children: the need for personalised medicine for children with rare neurological diseases}

In the European Union (EU), a rare disease is defined as one that affects fewer than five people per 10,000 [33]. Most of these conditions are genetically inherited and, importantly, many develop during childhood. Although individually uncommon, collectively there are thousands of rare diseases, making them an important group of disorders that affect a large number of patients throughout Europe. In the context that we have so far identified somewhere in the region of 5,000-8,000 distinct rare diseases, between 27 and 36 million people in the EU could be affected (6\%-8\% of the population), making the management of these conditions an important issue [33]. For these reasons, the EU takes the position that rare diseases are a serious public health concern and should be a priority in EU health and research programmes.

Although neurodegenerative diseases are most prevalent in the elderly, in rare cases they also affect individuals early in life. In children, neurodegeneration leads to severe mental retardation and premature death with devastating consequences on their immediate environment. These conditions also have relatively high costs for society. In total, there are thought to be about 600 different genetically inherited metabolic diseases, approximately 400 of which involve the CNS with differing degrees of severity and rates of degeneration. Lysosomal storage diseases (LSDs) are a subgroup of approximately 40 of these metabolic disorders that affect about one in 7,500 newborns [34, 35], although more recently a combined incidence as high as one in 5,000 newborns has been suggested [36]. The proportion of patients diagnosed with different LSDs in Australia between 1980 and 1996 is shown in Fig. 1. These conditions are caused by the lack of certain (lysosomal) enzymes or lysosome components, thus preventing the complete degradation of macromolecules and the recycling of their components. As a result of the failure to degrade these molecules, intermediate degradation products begin to accumulate within the cells, affecting the function of lysosomes and other cellular organelles. Each disorder is named based on the type of protein molecules that accumulate. The accumulation of these proteins starts immediately after birth and continues throughout life, often altering the structure and impairing the function of several organs, including the CNS (Fig. 2). CNS pathology causes mental retardation and progressive neurodegeneration that ultimately ends in early death of these young patients [37-41].

Our understanding of the pathogenesis of the LSDs has grown dramatically over the last $10-20$ years and there is already a strong community supporting research and scientific developments in this area, which includes physicians, researchers, patient advocates and industry. Thanks largely to advances in cellular and molecular biology, and the incentives for drug development provided by the introduction of the Orphan Drug Regulation in Europe, we are now moving closer to being able to provide therapies for neurological signs and symptoms in patients with these conditions. The advanced state of knowledge of the mechanisms of action of LSDs, in particular, is allowing the elucidation of features of neurodegenerative diseases that will most likely also prove to be useful in the study of more prevalent neurodegenerative diseases that predominantly affect the elderly (e.g. Alzheimer's and Parkinson's diseases). Furthermore, secondary events that lead to 


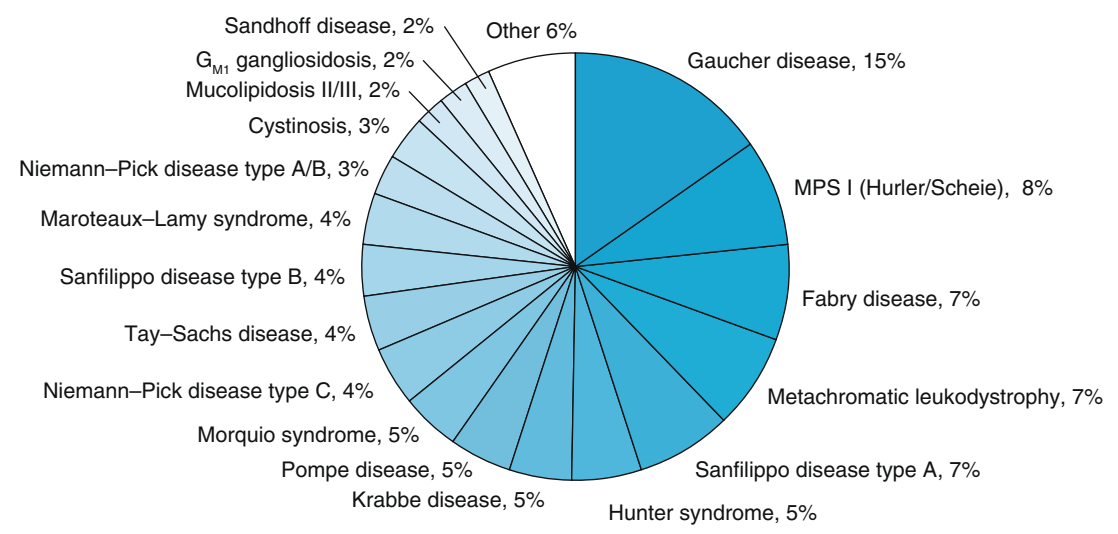

Fig. 1 Proportion of patients diagnosed with different lysosomal storage diseases (LSDs) in Australia between 1980 and 1996. More than ten patients were identified for 18 of the 27 LSDs diagnosed

during this period. Based on data from Meikle et al. [35]. MPS I, mucopolysaccharidosis type I
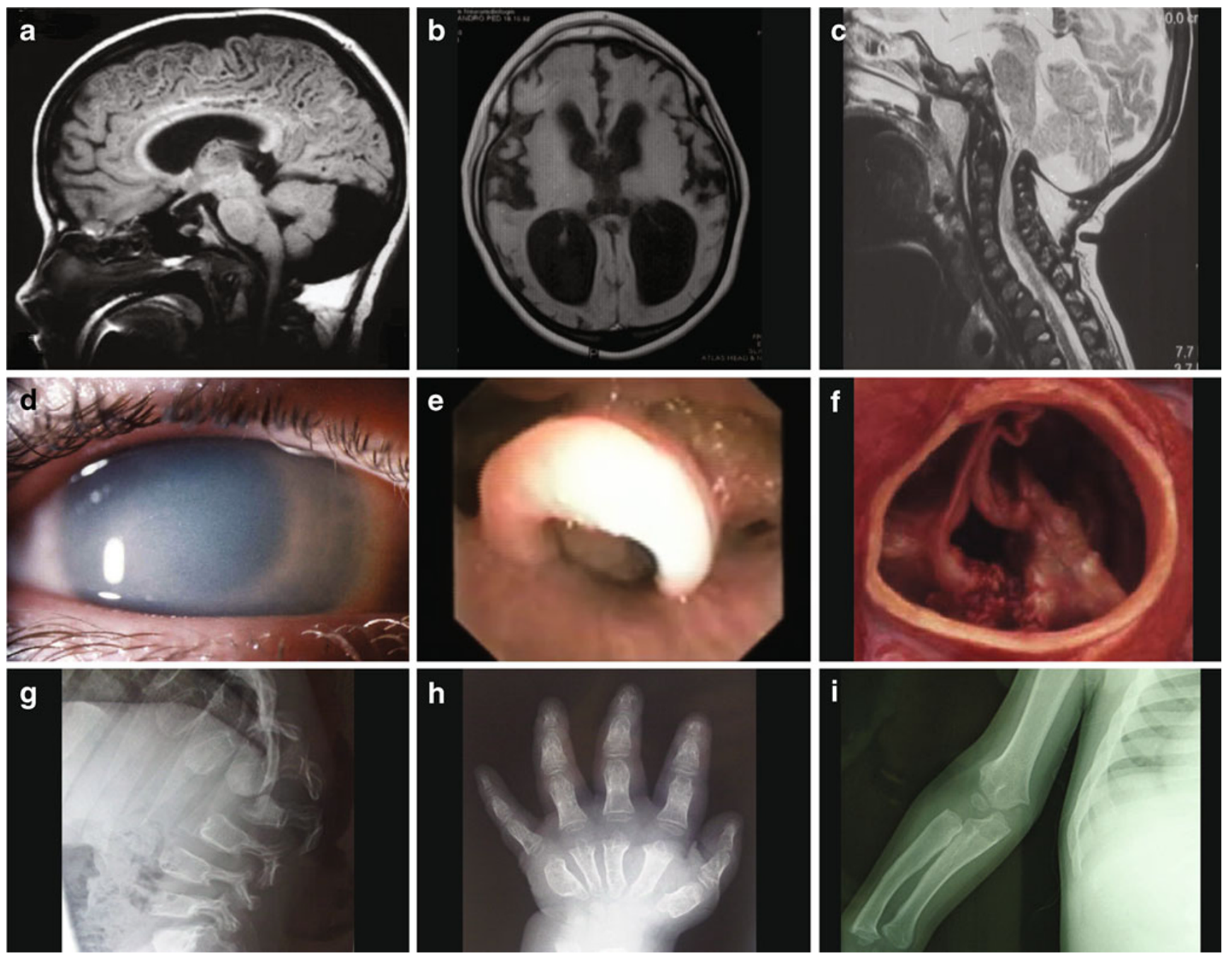

Fig. 2 Impact of the accumulation of undegraded proteins in lysosomal storage diseases. Images include brain scans showing: a atrophy with enlarged Virchow-Robin spaces and ventricles, b communicating hydrocephalus, and c spinal cord compression at

the cervical junctions. Other images show $\mathbf{d}$ corneal clouding, $\mathbf{e}$ larynx thickening, $\mathbf{f}$ valvular alterations due to the accumulation of undegraded substrates, and $\mathbf{g}-\mathbf{i}$ dysostosis multiplex of back bone, hand and long bones 
neurodegeneration (e.g. brain inflammation, alteration of intracellular trafficking, impairment of autophagy, or oxidative stress) are common to both paediatric and adult neurodegenerative diseases (for a review see [40]) Therefore, at least some of the observations relative to loss and recovery of brain plasticity in paediatric neurodegeneration can be extrapolated to adult disorders, and findings will provide unique insights into the pathophysiology and restorative capacities of neurodegenerative diseases in general. In fact, suppressing the primary cause of neurodegeneration (e.g. by supplementing the missing enzyme) in a young brain has the potential to maximise benefit as the brain retains considerable plasticity at this stage of development.

There are several factors that make the LSDs good candidates for pharmacogenetic studies and personalised medicine. The first is that the genetic basis of most of these conditions is well defined; they are monogenic disorders and many disease-causing mutations have already been characterised. Although these mutations are often private, they all result in reduced enzyme levels and storage to varying degrees. Despite a current general lack of awareness of these conditions among physicians which can lead to delayed diagnosis, there is the potential for pre-symptomatic diagnosis and, in many cases, prenatal diagnosis [42-44]. This means that once biomarkers that predict therapeutic response have been identified, it will be possible to initiate the appropriate treatment at an early stage of disease. Furthermore, for many of the LSDs there is some overlap in the pathways that are involved in the degradation of the proteins that accumulate, making it likely that any biomarkers discovered may apply in more than one condition. Another factor is that patients show considerable phenotypic variability (even those with the same underlying genetic mutation) and there is similarly variation between individuals in terms of response to therapy. At present, many different therapeutic approaches have been proposed including replacement of the missing enzyme (enzyme replacement therapy, ERT), reduction of levels of the enzyme substrate (substrate reduction therapy, SRT), the use of chemical chaperones to stabilise existing but unstable enzyme (chaperone therapy), bone marrow transplantation, and gene therapies [45-47] (Fig. 3). Based on current knowledge, predicting which patients will respond to which treatment remains a challenge [48].

In order to make personalised medicine for children with rare neurological disorders a reality it is necessary to refine the techniques used to ensure that therapy gains access to the CNS. Although it is known which enzyme deficiency causes each of the LSDs, supplying a correct version of the affected lysosomal enzyme intravenously only allows for

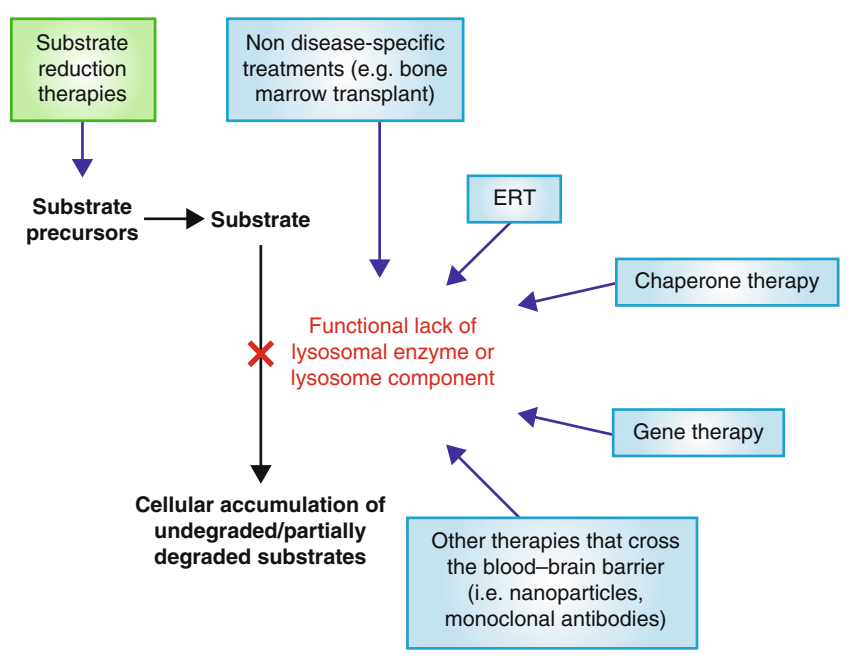

Fig. 3 Routes of intervention for the treatment of patients with lysosomal storage diseases

modification of the natural history of these diseases in the peripheral organs (e.g. liver, spleen, joints, etc.). The reason for this is that the therapeutic enzyme molecules used in ERT are too large to cross the blood-brain-barrier which protects the brain by allowing only those molecules required naturally by the CNS to pass into the brain from the peripheral circulation.

Proposed methods to circumvent the blood-brain-barrier in patients with LSDs include the direct injection into the brain of either the recombinant enzyme itself or of gene transfer vehicles able to induce the synthesis of the enzyme in genetically deficient brain cells [49]. Another approach is to inhibit enzymes early in the biosynthetic pathway of the accumulating protein(s) to reduce the number of molecules that require degradation in the lysosome. Small pharmaceutical molecules that are capable of crossing the bloodbrain barrier are used to achieve this. In cases where the affected lysosomal enzyme is present but does not function because it is unstable, small pharmaceutical molecules known as chemical chaperones that can cross the bloodbrain-barrier may be used to stabilise the enzyme, thus at least partially restoring its function. As the process of neurodegeneration observed in LSDs is characterised by secondary events such as neuroinflammation, glutamatergic activation and oxidative stress, the use of small molecules with anti-inflammatory and/or neuroprotective properties may also be a useful adjunctive approach to treat LSDs. To date, the utility of most of these approaches has already been demonstrated in animal models.

Looking to the future, there is also a need to establish and validate biomarkers that will allow us to understand both disease pathophysiology and which patients are most likely to respond to the different therapies that are available. 
Although we are currently able to quantify the stored protein material and to assess changes in storage in response to treatment, in general, these variables have not proved to be reliable biomarkers for the LSDs. Other candidate biomarkers are already under investigation. In the future, the availability of biomarkers other than the stored material, will allow the choice of the most appropriate therapy (ERT, chaperone therapy or SRT) when taking into account patient phenotype and expected disease severity and course of progression. Once a therapy is developed that can slow or prevent the progression of neurodegeneration, it will become extremely important to facilitate early detection of the genetic defect by newborn screening to enable early intervention.

The development of new tools/technologies to diagnose patients and to predict disease severity and progression, as well as to assess new therapies, can be achieved only through collaboration between existing stakeholders. The first steps towards establishing a community to focus efforts on developing therapies for rare neurological diseases of childhood were taken in December 2010 at a meeting held at the European Parliament in Brussels. This meeting, which was organised by the Brains for Brain Foundation [B4B (www.brains4brain.eu)], the European Brain Council (EBC), the Lysosomal Storage Disease (LSD) Patient Collaborative and the Veneto Region, and sponsored by Member of the European Parliament, Mrs Amalia Sartori (Veneto, Italy), brought together stakeholders from 13 EU countries, as well as the USA and Brazil. Those registered for the meeting included 22 political representatives and members of 17 scientific organisations, 14 European family organisations, 10 biotechnology companies and over 40 European Universities. The organisers called for a new initiative to coordinate the efforts of all existing groups in Europe with the shared goal of improving the treatment and care of patients with rare neurological disorders. As commented in a recent editorial, there is a need for harmonised interaction between all stakeholders to enable the further development of personalised medicine [50]. In line with this, the present project will unite all interested parties, who are working to increase the visibility, recognition and awareness of rare neurological disorders in order to facilitate early diagnosis of these conditions; to promote and facilitate partnership and collaboration between physicians, researchers, patient advocates, carers, policy- and decision-makers and industry; to encourage and support research and the translation of scientific breakthroughs into clinical practice; to contribute to the establishment of a standard of care for patients with rare neurological disorders which is agreed across Europe; and to ensure equity of access to diagnosis, treatment and care.

\section{Concluding remarks and outlook}

Achieving better and more predictable treatment outcomes offers benefits in terms of reducing unnecessary treatment and side effects and may ultimately lead to more costeffective healthcare. In order to progress the development of personalised medicine it is necessary for these benefits to be clearly demonstrated and for there to be buy-in and investment from all stakeholders, including the pharmaceutical industry, regulatory authorities and payers. The increasing incorporation of biomarkers within product pipelines is a sign of the growing interest of the pharmaceutical industry in this area. Nevertheless, there is still work to do in order to realise the potential of this approach and there remains a need for more examples that demonstrate proof of concept in clinical practice.

Acknowledgements The authors would like to thank all of the members of the Brains for Brain Foundation and in particular the patients and families affected by lysosomal storage diseases.

Medical writing support to the authors during the preparation of the report was provided by Harriet Crofts, $\mathrm{PhD}$, of Oxford PharmaGenesis Ltd and was sponsored by Shire Human Genetic Therapies (Shire HGT). The sponsor had no influence on the contents or direction of the manuscript. The authors take full responsibility for the content of the paper.

Conflict of interest Maurizio Scarpa has received research grants and honoraria and travel support for speaking engagements from Actelion, Shire Human Genetic Therapies, Genzyme Corporation and BioMarin. David Begley has received research grants and honoraria and travel support for speaking engagements from Actelion, Shire Human Genetic Therapies, Genzyme Corporation, BioMarin and GlaxoSmithKline. Adriana Ceci, Rosella Tomanin and Pierpaolo Mincarone have no conflicts of interests.

\section{References}

1. Directive 2001/20/EC of the European Parliament and of the Council of 4 April 2001 on the approximation of the laws, regulations and administrative provisions of the Member States relating to the implementation of good clinical practice in the conduct of clinical trials on medicinal products for human use. Official Journal of the European Commmunities. 2001;L121.

2. Commission directive 2005/28/EC of 8 April 2005 laying down principles and detailed guidelines for good clinical practice as regards investigational medicinal products for human use, as well as the requirements for authorisation of the manufacturing or importation of such products. Official Journal of the European Commmunities. 2005; L91.

3. Regulation (EC) No 726/2004 of the European Parliament and of the Council of 31 March 2004 laying down Community procedures for the authorisation and supervision of medicinal products for human and veterinary use and establishing a European Medicines Agency. Official Journal of the European Union. 2004;L136.

4. Hung SI, Chung WH, Jee SH, Chen WC, Chang YT, Lee WR, et al. Genetic susceptibility to carbamazepine-induced cutaneous 
adverse drug reactions. Pharmacogenet Genomics. 2006;16:297306.

5. Hetherington S, Hughes AR, Mosteller M, Shortino D, Baker KL, Spreen W, et al. Genetic variations in HLA-B region and hypersensitivity reactions to abacavir. Lancet. 2002;359:1121-2.

6. Kauf TL, Farkouh RA, Earnshaw SR, Watson ME, Maroudas P, Chambers MG. Economic efficiency of genetic screening to inform the use of abacavir sulfate in the treatment of HIV. Pharmacoeconomics. 2010;28:1025-39.

7. Chung WH, Hung SI, Chen YT. Genetic predisposition of lifethreatening antiepileptic-induced skin reactions. Expert Opin Drug Saf. 2010;9:15-21.

8. Vogenberg FR, Isaacson Barash C, Pursel M. Personalized medicine: part 1: evolution and development into theranostics. Pharmacy \& Therapeutics 2010;35:560-76.

9. Loo TT, Ross CJ, Sistonen J, Visscher H, Madadi P, Koren G, et al. Pharmacogenomics and active surveillance for serious adverse drug reactions in children. Pharmacogenomics. 2010;11:1269-85.

10. Ceci A, Felisi M, Baiardi P, Bonifazi F, Catapano M, Giaquinto C, et al. Medicines for children licensed by the European Medicines Agency (EMEA): the balance after 10 years. Eur J Clin Pharmacol. 2006;62:947-52.

11. Leeder JS, Kearns GL, Spielberg SP, van den Anker J. Understanding the relative roles of pharmacogenetics and ontogeny in pediatric drug development and regulatory science. J Clin Pharmacol. 2010;50:1377-87.

12. Kearns GL, Abdel-Rahman SM, Alander SW, Blowey DL, Leeder JS, Kauffman RE. Developmental pharmacology-drug disposition, action, and therapy in infants and children. N Engl J Med. 2003;349:1157-67.

13. Strassburg CP, Strassburg A, Kneip S, Barut A, Tukey RH, Rodeck B, et al. Developmental aspects of human hepatic drug glucuronidation in young children and adults. Gut. 2002;50:25965 .

14. Odell GB. "Physiologic" hyperbilirubinemia in the neonatal period. N Engl J Med. 1967;277:193-5.

15. Kawade N, Onishi S. The prenatal and postnatal development of UDP-glucuronyltransferase activity towards bilirubin and the effect of premature birth on this activity in the human liver. Biochem J. 1981;196:257-60.

16. Miyagi SJ, Collier AC. Pediatric development of glucuronidation: the ontogeny of hepatic UGT1A4. Drug Metab Dispos. 2007;35:1587-92.

17. Rodriguez W, Selen A, Avant D, Chaurasia C, Crescenzi T, Gieser $\mathrm{G}$, et al. Improving pediatric dosing through pediatric initiatives: what we have learned. Pediatrics. 2008;121:530-9.

18. Paolucci P, Jones KP, del Carmen Cano Garcinuno M, Catapano $\mathrm{M}$, Iolascon A, Ceci A. Challenges in prescribing drugs for children with cancer. Lancet Oncol. 2008;9:176-83.

19. Russo R, Capasso M, Paolucci P, Iolascon A. Pediatric pharmacogenetic and pharmacogenomic studies: the current state and future perspectives. Eur J Clin Pharmacol. 2010. doi:10.1007/ s00228-010-0931-1.

20. Krekels E, Ceci A, Iolascon A, Girotto S, Della Pasqua O. The role of paediatric pharmacogenetic studies in Europe. Pharmaceutical Policy and Law. 2009;11:23-30.

21. Holmes MV, Shah T, Vickery C, Smeeth L, Hingorani AD, Casas JP. Fulfilling the promise of personalized medicine? Systematic review and field synopsis of pharmacogenetic studies. PLoS ONE. 2009;4:e7960.

22. Becker ML, Leeder JS. Developmental pharmacogenetics in pediatric rheumatology: utilizing a new paradigm to effectively treat patients with juvenile idiopathic arthritis with methotrexate. Hum Genomics Proteomics. 2010. doi:10.4061/2010/257120.

23. Arnold D, Jones BL. Personalized medicine: a pediatric perspective. Curr Allergy Asthma Rep. 2009;9:426-32.
24. Carroll WL, Bhojwani D, Min DJ, Raetz E, Relling M, Davies S, Downing JR, Willman CL, Reed JC. Pediatric acute lymphoblastic leukemia. Hematology Am Soc Hematol Educ Program. 2003:102-31.

25. Yates CR, Krynetski EY, Loennechen T, Fessing MY, Tai HL, Pui $\mathrm{CH}$, et al. Molecular diagnosis of thiopurine S-methyltransferase deficiency: genetic basis for azathioprine and mercaptopurine intolerance. Ann Intern Med. 1997;126:608-14.

26. Lennard L, Lilleyman JS, Van Loon J, Weinshilboum RM. Genetic variation in response to 6-mercaptopurine for childhood acute lymphoblastic leukaemia. Lancet. 1990;336:225-9.

27. Relling MV, Hancock ML, Rivera GK, Sandlund JT, Ribeiro RC, Krynetski EY, et al. Mercaptopurine therapy intolerance and heterozygosity at the thiopurine S-methyltransferase gene locus. J Natl Cancer Inst. 1999;91:2001-8.

28. Relling MV, Gardner EE, Sandborn WJ, Schmiegelow K, Pui CH, Yee SW, et al. Clinical pharmacogenetics implementation consortium guidelines for thiopurine methyltransferase genotype and thiopurine dosing. Clin Pharmacol Ther. 2011;89:387-91.

29. Carleton B, Poole R, Smith M, Leeder J, Ghannadan R, Ross C, et al. Adverse drug reaction active surveillance: developing a national network in Canada's children's hospitals. Pharmacoepidemiol Drug Saf. 2009;18:713-21.

30. Madadi P, Ross CJ, Hayden MR, Carleton BC, Gaedigk A, Leeder JS, et al. Pharmacogenetics of neonatal opioid toxicity following maternal use of codeine during breastfeeding: a case-control study. Clin Pharmacol Ther. 2009;85:31-5.

31. Crowley JJ, Sullivan PF, McLeod HL. Pharmacogenomic genome-wide association studies: lessons learned thus far. Pharmacogenomics. 2009;10:161-3.

32. November/December Tufts CSDD Impact Report 2010.

33. Council recommendation on action in the field of rare diseases, 2009. http://www.consilium.europa.eu/uedocs/cms data/docs/ pressdata/en/lsa/108383.pdf (Accessed [11 Nov 2010]).

34. Poorthuis BJ, Wevers RA, Kleijer WJ, Groener JE, de Jong JG, van Weely S, et al. The frequency of lysosomal storage diseases in The Netherlands. Hum Genet. 1999;105:151-6.

35. Meikle PJ, Hopwood JJ, Clague AE, Carey WF. Prevalence of lysosomal storage disorders. JAMA. 1999;281:249-54.

36. Fuller M, Meikle PJ, Hopwood JJ. Epidemiology of lysosomal storage diseases: an overview. In: Mehta A, Beck M, SunderPlassmann $\mathrm{G}$, editors. Fabry disease: perspectives from 5 years of FOS. Oxford: Oxford PharmaGenesis Ltd; 2006. p. 9-20.

37. Neufeld EF, Muenzer J. The mucopolysaccharidoses. In: Scriver CR, Beaudet AL, Sly WS, Valle D, editors. The metabolic and molecular bases of inherited disease. New York: McGraw-Hill; 2001. p. 3421-52.

38. Gieselmann V, Krageloh-Mann I. Metachromatic leukodystrophyan update. Neuropediatrics. 2010;41:1-6.

39. Parkinson-Lawrence EJ, Shandala T, Prodoehl M, Plew R, Borlace GN, Brooks DA. Lysosomal storage disease: revealing lysosomal function and physiology. Physiology (Bethesda). 2010;25:102-15.

40. Bellettato CM, Scarpa M. Pathophysiology of neuropathic lysosomal storage disorders. J Inherit Metab Dis. 2010;33:34762.

41. Vitner EB, Platt FM, Futerman AH. Common and uncommon pathogenic cascades in lysosomal storage diseases. J Biol Chem. 2010;285:20423-7.

42. Duffner PK, Caggana M, Orsini JJ, Wenger DA, Patterson MC, Crosley CJ, et al. Newborn screening for Krabbe disease: the New York State model. Pediatr Neurol. 2009;40:245-52.

43. Schroder W, Petruschka L, Wehnert M, Zschiesche M, Seidlitz G, Hopwood JJ, et al. Carrier detection of Hunter syndrome (MPS II) by biochemical and DNA techniques in families at risk. J Med Genet. 1993;30:210-3. 
44. Meikle PJ, Grasby DJ, Dean CJ, Lang DL, Bockmann M, Whittle AM, et al. Newborn screening for lysosomal storage disorders. Mol Genet Metab. 2006;88:307-14.

45. Kaye EM. Lysosomal storage diseases. 2001;3:249-56.

46. Anson DS, McIntyre C, Byers S. Therapies for neurological disease in the mucopolysaccharidoses. Curr Gene Ther. 2011;11:132-43.

47. Neufeld EF. Enzyme replacement therapy - a brief history. In: Mehta A, Beck M, Sunder-Plassmann G, editors. Fabry disease: perspectives from 5 years of FOS. Oxford: Oxford PharmaGenesis Ltd; 2006. p. 93-9.

48. Escolar ML, Poe MD, Martin HR, Kurtzberg J. A staging system for infantile Krabbe disease to predict outcome after unrelated umbilical cord blood transplantation. Pediatrics. 2006;118:e879-89.

49. Begley DJ, Pontikis CC, Scarpa M. Lysosomal storage diseases and the blood-brain barrier. Curr Pharm Des. 2008;14:1566-80.

50. Golubnitschaja O. Promoting predictive, preventive and personalised medicine in global scale. EPMA J. 2010;1:523-4. 